\title{
La traduction comme engagement : René R. Khawam et Jamel Eddine Bencheikh, passeurs de la littérature arabe
}

Résumé: Traducteurs et commentateurs de la poésie et de la narration arabes, dont Les Mille et Une Nuits, René Khawam et Jamel Eddine Bencheikh ont tous les deux œuvré à une meilleure connaissance et à une réévaluation de cette littérature. Bien que ces deux passeurs contemporains l'un de l'autre aient effectué des choix différents dans leur approche philologique du texte, ils ont mis en valeur la liberté de parole subversive d'auteurs et de textes inconnus ou mal-connus en France, intégrés ou marginaux par rapport aux lettres arabes. Avec ces œuvres, ils ont cherché à explorer un imaginaire « contrôlé » par les érudits des deux rives et les stéréotypes orientalistes. En plus de la traduction, la glose, la vulgarisation et la création littéraire ont porté leur engagement à changer le regard non seulement sur la littérature mais aussi sur la culture et la société arabo-musulmanes.

Mots clés: Traduction, littérature arabe, Mille et Une Nuits, dogme, poésie

Alors que la France avait dû attendre près de deux siècles sa deuxième traduction des Mille et Une Nuits, par Joseph-Charles Mardrus, après celle fondatrice d'Antoine Galland, la fin du XXe siècle vit deux autres traductions, à peu de temps d'intervalle, prétendre à un renouvellement de la lecture de cette œuvre, la plus célèbre de la littérature arabe.

René Rizqallah Khawam proposa une première version en 1965-1967 puis la réédita, modifiée, en 1985-1986 chez Phébus. Dans ce même intervalle, Jamel Eddine Bencheikh et André Miquel commencèrent à publier une série d'études sur les Nuits ainsi que la traduction de contes choisis en 4 volumes dans la collection Folio de Gallimard. Ce travail ne constitua que la première étape d'une traduction critique « complète » parue de 2005 à 2007 dans la prestigieuse « Bibliothèque de la Pléiade » du même éditeur. Toutes les deux ambitieuses - bien que ne bénéficiant pas de la même valeur éditoriale -, ces traductions concurrentes divergent dans leur approche. Celle de René Khawam se réclame d'une authenticité qui, à la différence de celle affichée par la version de Joseph Charles Mardrus au début du siècle ${ }^{1}$,

1 La traduction de Mardrus, publiée entre 1899 et 1904, a suscité l'enthousiasme de la part des écrivains et artistes de l'époque et a connu un très large succès, à tel point qu'elle a relancé la

Ә Open Access. $\odot 2020$ Cyrille François, published by De Gruyter. (C) BYr-Nc-ND This work is licensed under a Creative Commons Attribution-NonCommercial-NoDerivatives 4.0 License. 
cherchait à séparer le bon grain de l'ivraie et, ce faisant, restreint le corpus des contes. Celle de Bencheikh et Miquel, à l'inverse, conçoit la compilation comme un trésor enrichi par la diversité des apports.

En 1985 toujours, parallèlement à sa " réhabilitation » des « vraies » Mille et Une Nuits, René Khawam publie, presque trente ans après André Miquel, une nouvelle traduction en français de la traduction arabe par Ibn al-Muqaffâ' au VIII ${ }^{\mathrm{e}}$ siècle du Kalîla et Dimna indo-persan. Dans l'introduction, présentant la réception du texte d'Ibn al-Muqaffâ' par le milieu intellectuel et politique des Abbassides, il dénonce l'apparition rapide de traductions arabes fallacieuses visant à contester celle du premier traducteur : « Ce procédé [...] consiste à lancer, à grand renfort de publicité, de nouvelles fausses traductions des Mille et Une Nuits, afin de faire oublier l'authentique, faite sur les manuscrits. » (Ibn al-Muqaffâ' 1985, 26). Bien qu'il ne désigne explicitement personne, cette remarque pourrait viser le projet de traduction fragmentaire des Mille et Une Nuits par André Miquel et Jamel Eddine Bencheikh.

En dépit de leur divergence philologique - sur laquelle nous reviendrons -, les deux traducteurs partagent plusieurs points communs en plus de leur passion pour la transmission de la littérature arabe. L'un et l'autre étaient de bons, voire d'excellents connaisseurs de la poésie arabe, en particulier classique. René Khawam a colligé une anthologie de poésie, éditée par Phébus en 1995, dans laquelle la poésie classique tient une bonne part. Jamel Eddine Bencheikh, quant à lui, dans sa Poétique arabe en 1975, a subverti l'approche traditionnelle de la poésie classique du III ${ }^{\mathrm{e}}$ siècle de l'hégire / IX ${ }^{\mathrm{e}}$ siècle ap. J.-C. en appliquant une perspective à la fois sociocritique et structuraliste, c'est-à-dire en revisitant par une méthodologie moderne occidentale un canon des lettres arabes que la culture savante a transmis, protégé, reproduit comme un modèle incontournable.

Outre la poésie, tous les deux ont centré leurs travaux sur la narration et ce que l'on appelle « les écritures de l'imaginaire ", avec Les Mille et Une Nuits en commun. Dans ce domaine en particulier, tous deux font preuve d'un engagement culturel quant à la sélection des textes parmi le patrimoine littéraire arabe tant pour leur statut que pour leurs contenus idéologiques.

Dans ce qui suit, nous présenterons cet engagement en faveur d'un nouveau regard sur la littérature arabe qui passe, pour l'un, par une philologie centrée sur la singularité auctoriale et textuelle et, pour l'autre, par une investigation de l'imaginaire collectif.

mode des Mille et Une Nuits et de l'exotisme oriental. Grâce à cela, elle s'est imposée comme la deuxième traduction française majeure avec celle, pionnière, d'Antoine Galland (1704-1717). 


\section{Un autre regard sur le canon littéraire}

« Dénicheur de textes » comme l'appelle Sylvette Larzul, René Khawam² a multiplié les traductions d'œuvres importantes au regard de la littérature arabe classique et post-classique, comme Les aventures de Kalîla et Dimna (1985), les Séances [al-Maqāmāt] d'Harîrî (texte datant de 1257), un roman du Libanais Faris Chidyak, un des fondateurs du roman moderne, mais également des œuvres en marge du canon littéraire, moins bien considérées par les lettrés, comme le théâtre d'ombre d’Ibn Dâniyâl, en 1997.

Son anthologie de la poésie arabe (1995 [1977]) dresse le panorama d'une poésie si prestigieuse qu'elle incarne à elle seule la fine fleur prestigieuse de la littérature. Comme pour chacune de ses traductions, Khawam veut légitimer son travail par opposition à celui des prédécesseurs. C’est pourquoi il déclare que sa poétique privilégie l'allusif à l'évident, l'esprit de la langue arabe, le souffle qui l'emplit, à son ancrage référentiel :

\footnotetext{
Qu'on veuille ne considérer que les mots en les classant d'après la racine, les formes verbales, le sens « évident », on n'obtiendra de l'œuvre qu'un relevé : sa réalité secrète - essentielle - échappera. Qu'on ne s'étonne pas, à ce compte, de voir tant de traductions s'arrêter à la signification la plus étroite du poème, laissant fuir entre les mots de la langue qui le transpose la précieuse ambiguïté de sa poésie. (Khawam 1995 [1977], 15)
}

Il recentre donc l'attention sur le matériau de la poésie, la chair du verbe. À un premier stade, il projette de réhabiliter la vérité, ailleurs travestie, de la langue arabe. Or, cette défense de la langue et de la littérature dépasse le poétique pour se porter vers le philologique et l'idéologique, l'inspection et la critique de la réception.

L'exemple de Kalîla et Dimna donne une idée du combat que Khawam mène contre les lettrés arabes et les traducteurs des diverses langues impliquées dans la transmission de l'œuvre. Dans l'introduction à sa traduction, il pointe la censure exercée par des modifications ou la présentation sous forme d'extraits à cause de propos embarrassant les instances du pouvoir ou certains intellectuels.

2 Né à Alep en 1917 dans une famille chrétienne lettrée originaire du Karabagh, René Rizqallah Khawam étudia à l'Université Catholique de Beyrouth, qui formait une élite maronite bilingue et biculturelle censée contribuer à l'administration de la colonie. Il s'installa à Paris en 1947, où il passa des diplômes d'histoire, de géographie, de psychologie, de littérature française et de linguistique générale. Il suivit aussi des cours de littérature arabe et devint un disciple et un proche de Louis Massignon. De 1958 à 1982, il poursuivit sa carrière de professeur d'histoire à l'École Massillon, à Paris. Quand fut fondée en 1976 la maison d'édition Phébus, il y était chargé du « Domaine arabe », qu'il dirigea jusqu'en 1998. 
Ce qui fait que le livre de Kalila et Dimna d'Ibn al-Mouqaffa', l'authentique, est une synthèse entre la pensée arabe anté-islamique, islamique du premier siècle de l'Islam, donc omayyade [...] et l'apport indien, iranien, grec, araméen. Cela ne concordait pas avec la nouvelle politique de Baghdâd et celle de certains juristes influents. Il fallait supprimer cette preuve de la vitalité de la pensée arabe et bédouine en milieu pré-islamique et omayyade. Seul moyen possible : escamoter les preuves introduisant ces citations [...], présenter tout le livre comme une traduction de l'indien par l'intermédiaire du persan, faire prévaloir l'influence iranienne. (Ibn al-Muqaffa' 1985, 27)

Se plaisant à moquer les bien-pensants, vilipendant les « cagots » soumis au pouvoir et cultivant un certain esprit de désobéissance, Khawam s'en prend dès que possible aux lettrés, cheikhs ou écrivains, instrumentalisés par l'idéologie moralisatrice dominante - ce que Bencheikh appelle « la Loi » - qui s'efforce de contrer l'art d'écrivains comme al-Souyoûti ou al-Harîrî. Le lecteur comprend alors mieux la mission qui incombe au traducteur : défaire ces textes de la pruderie qui entraverait leur juste transmission.

Ce silence embarrassant, nos contemporains l'ont hérité des écrivains arabes du milieu canoniste du XVIII siècle, à l'époque où s'est amorcée la renaissance qui s'épanouit aujourd'hui en redonnant de beaux chefs-d'œuvre. Pour retrouver la saine liberté de l'expression, il faut remonter aux auteurs du XVII ${ }^{\mathrm{e}}$ siècle, qui ne craignaient pas de nommer les choses par leurs noms, selon la bonne tradition arabe. (al-Qâsîm al-Harîrî 1992, 9)

Les censeurs arabes n'ont pas l'exclusivité de ses critiques : s'ils obéissent à d'autres motivations, les orientalistes européens participent à la distorsion de la transmission en écartant cette crudité ou, au contraire, en l'exagérant. En conséquence, il s'agit pour le traducteur de s'émanciper de la chaîne de transmission des lettrés pour revenir à des manuscrits " originaux », dans le cas des œuvres déjà traduites, ou pour offrir la primeur d'une œuvre encore jamais traduite.

Mettant régulièrement l'accent sur son effort d'exhumation des manuscrits ignorés, Khawam pratique une philologie soucieuse d'établir la paternité de chaque texte - démarche qui s'avère source de polémique quand il s'agit des Mille et Une Nuits, mais qui, dans le cas de Kalîla et Dimna, a pour mérite d'évaluer la part de créativité dans la traduction d'un auteur. Plus que Jamel Eddine Bencheikh, il s'est employé à réhabiliter des textes ignorés jusqu'ici par la littérature française, notamment Désir de femmes. À propos de ce dernier ouvrage, il souligne une approche subversive par la place qu'Hawrânî donne aux femmes : «il leur ouvre une tribune, leur souffle des revendications, leur distribue des armes. " (Hawrânî 1994, 14). De même, dans l'introduction à La Prairie des gazelles, il met l'accent sur le pouvoir que les femmes détiennent bien qu'elles soient « officiellement reléguée au fond de leurs appartements » (Nawâiî 1989, 11). Le traducteur se refuse pourtant à n'être que le médiateur du passé littéraire. 
Afin de montrer que les textes qu'il exhume sont profitables au lecteur, Khawam pointe ce en quoi ils fournissent

\begin{abstract}
plus d'actualité - ou plus de richesses à même de nourrir notre actualité - que [ce qu'on trouve] dans bien des écrits modernes supposés s'adresser à leur siècle. Témoin le livre [Le désir des femmes] qu'on va lire, qui malgré ses six cents ans possède [...] surtout une libre façon d'aborder telles questions dont nous n'avons toujours pas fait le tour, une indépendance de ton et de propos que notre époque, nous semble-t-il, gagnerait à méditer. (Hawrânî 1994, 9)
\end{abstract}

C'est grâce aux textes érotiques, comme le célèbre La Prairie parfumée où s'ébattent les plaisirs d'al-Nafzâwî, que Khawam récupère la tradition arabe et libertaire du franc-parler. La présentation qu'il fait des Délices des cours ou Ce que l'on ne trouve en aucun livre d'al-Tîfâchî, en 1971, ou de La Prairie des gazelles : Éloge des beaux adolescents de Mohammad al-Nawâdji (début XVe siècle), en 1989, conduit à bouleverser l'image sulfureuse que l'on peut accoler aux auteurs de ces ouvrages. En effet, à rebours d'une conception simpliste qui opposerait des francs-tireurs libertins aux écrivains moralistes, les marginaux aux classiques, les auteurs érotologues qu'il présente peuvent combiner ouvrages religieux et érotiques, comme Abd al-Rahmane al-Souyoûti (1445-1505), auteur des Nuits de noces, ou comment humer le doux breuvage de la magie licite, professeur spécialisé dans le droit et les traditions musulmanes et commentateur du Coran.

Beaucoup moins prolixe que Khawam - ce qui s'expliquerait en partie par ses fonctions d'enseignant-chercheur - Jamel Eddine Bencheikh a partagé l'attention de son confrère pour la poésie classique et l'expression du désir. S'il n'a pas publié d'anthologie de la littérature arabe classique - mais de la poésie algérienne francophone moderne -, il a offert une étude qui a fait date sur ce canon littéraire en y appliquant la distance d'une analyse structurale et sociologique : son objectif consistait

à partir de la production littéraire arabe la plus ancienne et la plus typique, [à] scruter l'horizon culturel d'une société ; à travers un langage, au-delà de l'abstraction, retrouver l'homme, comprendre son accord avec le monde. L’ambition désignait son objet, la poésie médiévale, et un premier objectif, les modes d'une création. Il lui fallait, pour atteindre l'un et saisir l'autre, une méthode et des moyens. (Bencheikh 1975, 3)

Universitaire, doté de la « [p]osture idéale du bilingue biculturel, quasi natif des deux langues à qui est dévolu, comme naturellement, ce privilège d'analyser les textes à partir de leur "extranéité intérieure" »(Khadda 1998, 34), Bencheikh ${ }^{3}$ a

3 Né à Casablanca en 1930 dans une famille originaire de Tlemcen en Algérie, Jamel Eddine Bencheikh s'initia et se forma à la littérature française par la lecture des poètes et écrivains fran- 
mis à profit à la fois le pas de côté que lui permet l'autre culture et la rigueur scientifique de sa formation pour apporter un autre regard sur la littérature arabe - doublement autre, pourrait-on dire, car différent du discours habituel des spécialistes arabes et des orientalistes européens. Pour reprendre les termes de Naget Khadda, « [c]'est là que réside originalité de son approche qui le distingue des héritiers du corpus et des orientalistes avec lesquels il peut partager le bagage critique mais pas la mémoire affective dont dépend aussi la réception d'une œuvre. " (Khadda 1998, 33).

Plusieurs années après cette Poétique arabe, la seconde grande entreprise scientifique de Bencheikh a porté sur Les Mille et Une Nuits, où l'on retrouve à nouveau ce regard renouvelé, fruit d'une collaboration de la tradition française des Nuits et d'une connaissance intime de la littérature arabe.

\section{Traduire Les Mille et Une Nuits : l'authenticité contre la libéralité}

La traduction des Mille et Une Nuits par René Khawam conteste avec véhémence une démarche « libérale » qui a consisté à joindre à la compilation, à partir du XVe siècle surtout, des récits empruntés à d'autres compilations, à d'autres traditions, à d'autres auteurs. À l'inverse de cette ouverture du corpus, Khawam défend l'idée selon laquelle Les Mille et Une Nuits sont l'œuvre d'un seul auteur, dont le manuscrit, probablement du XIII' ${ }^{\mathrm{e}}$ siècle, n'existerait plus, mais peut être reconstitué a posteriori grâce à une exploration critique des traditions manuscrites. Cette thèse est d'ailleurs similaire à celle qui prévaut dans sa traduction de Kalîla et Dîmna, dont le manuscrit d'origine a disparu et que l'on ne connaît que par des copies qu'il juge altérées, corrompues.

Tenant à rendre à chacun son dû, le traducteur regrette, dans une longue mise au point, qu'un des auteurs qu'il traduit par ailleurs, al-Hawrânî, ait été pillé au profit des Nuits :

çais : Paul Éluard, Louis Aragon, Albert Camus, Saint-John Perse, René Char, René Guy Cadou. Il quitta le Maroc en 1948 pour la France, puis l'Algérie. Agrégé d'arabe, il enseigna à l'Université d'Alger à partir de 1962, où il créera une section de Littérature comparée. À partir de 1969, il continua sa carrière universitaire à Paris, où il enseigna finalement à la Sorbonne jusqu'en 1997. Parallèlement à ses travaux scientifiques, il publia diverses chroniques politiques et littéraires en $\mathrm{Al}$ gérie - sensible et impliqué par les espoirs et les violences de son histoire. Il écrivit aussi des études d'œuvres d'arts (recueillies) et donna surtout une œuvre de poésie publiée à partir de 1981. 
Les Arabes eux-mêmes, qui ont puisé dans le corpus de l'admirable Hawrânî pour en farcir sans vergogne leur édition des Mille et Une Nuits (celle de Boulâq, réalisée en 1835 sous l'œil puritain des censeurs de l'université d'al-Azhar au Caire), n'ont pas hésité à mélanger à son récit celui d'un autre roman qui n'avait rien à voir avec lui, jusqu'à en tirer [...] un produit de synthèse tristement expurgé, résumé, souvent incohérent. Certes, c'était là rendre à son livre le plus bel hommage : celui de Schéhérazade. Mais c'était le trahir à en faire se retourner le pauvre auteur dans sa tombe. Galland dans son édition des Nuits (cent ans avant le massacre du texte perpétré à Boulâq) a bien sûr ignoré cet « emprunt »; mais Mardrus au siècle suivant l'a tranquillement repris à son compte, et après lui tous les autres traducteurs jusqu'aux plus récents - lesquels ne peuvent cependant ignorer là qu'ils utilisent là des sources qui n’ont aucun rapport avec le texte authentique des Nuits. (Hawrânî 1994, 13)

Khawam déblaie le corpus des Nuits afin d'en extraire tous les récits qui, selon lui, y ont été interpolés. Une des conséquences les plus éclatantes de cette réévaluation réside dans la publication séparée de ce qu'il titre Les Aventures de Sindbad le Marin et Le roman d'Aladdin. L'un et l'autre avaient été incorporés aux Nuits au tout début du XVIII siècle par Antoine Galland, dans ce qui fut à la fois la première traduction et la première édition mondiales. Rappelant cette hétérogénéité, Khawam brandit ce vice d'authenticité non seulement pour écarter de sa vraie version ces deux récits, mais aussi pour publier indépendamment les originaux de ces textes. Si cela ne pose aucune difficulté concernant Sindbad, dont Antoine Galland possédait effectivement un manuscrit indépendant des Nuits, on peut être plus dubitatif concernant Aladin. En effet, on sait que Galland a écrit le récit d'Aladin à partir de la narration orale d'un maronite d'Alep, Hanna Diap, qui s'est imposée comme la seule version disponible. Or, Khawam prétend recourir à deux manuscrits du XI ${ }^{e}$ siècle, présentés comme authentiques, offrant une leçon qui « diffère très largement du texte que donne Galland » (Khawam 1988, 8). Néanmoins, il n'apporte aucune information complémentaire sur ces manuscrits, se contentant de divulguer de bien maigres éléments pour justifier sa datation.

Cette démarche, rejoignant celles d'universitaires comme Nikita Elisseef (1915-1997), ne se légitime que par une reconstitution conjecturelle d'un texte authentique et entretient une certaine inexactitude quant à l'histoire des Nuits, bien plus complexe et incertaine que ce qu'il décrit. Le plus ancien manuscrit, celui que possédait le premier traducteur des Nuits, Antoine Galland, date du XVe siècle. On sait qu'il constitue la fixation par un auteur de traditions manuscrites antérieures, débutant, dans la littérature arabe, au VIII siècle, par la traduction en arabe d'un ouvrage persan. Rien ne justifie donc de déclarer une création singulière, pas plus aux alentours du XIII siècle qu'avant ou après. Au fur et à mesure de leur diffusion, les récits ont été transformés par des relais sans que l'on puisse attribuer à l'un d'entre eux la paternité d'un recueil qui serait authentique. Khawam, prenant acte des zones d'ombre de la génétique des Nuits, joue de la 
frontière entre " recueil d'une tradition »- qui suppose aussi qu'on y mette la patte en en faisant une création personnalisée - et œuvre singulière inspirée de nombreuses sources. Ceci dit, on peut cependant lui reconnaître le mérite de valoriser les Nuits comme une œuvre littéraire arabe, allant ainsi à rebours de l'idée selon laquelle ce sont des contes populaires qui n'ont atteint le rang d'œuvre que grâce aux traducteurs européens. En d'autres termes, Khawam réintègre Les Mille et Une Nuits dans la littérature arabe.

À l'inverse, l'impossibilité de décerner la palme de la perfection à aucune des versions conduit Jamel Eddine Bencheikh à défendre une conception intemporelle des Nuits : radicale, celle-ci distingue l'œuvre et sa matérialisation textuelle. Cette intemporalité est manifeste dans la mesure où, selon lui, elle conserverait un imaginaire ancien, préislamique qui dure malgré toutes les réécritures : «Étudier la langue, noter les variantes, etc., tout cela est toujours utile, mais sans efficacité sur un texte qui échappe à ce point à sa version. Celle - ou celles - que nous avons. Dix autres mises en forme sont possibles, qui revêtiront le même corps, mais n'épuiseront pas ses mystères. Le manuscrit d'un conte n'est qu'un moment de sa vie. " (Bencheikh 1988, 12)

La traduction qu'il a élaborée avec André Miquel n'entre pas dans le jeu de la plus haute authenticité. Au contraire, après avoir retracé l'histoire de la composition du « Trésor » et sa redécouverte au XVIII e siècle, puis la recherche des manuscrits au XIX ${ }^{\mathrm{e}}$ siècle, les deux traducteurs expliquent utiliser les versions de Bûlâq (Le Caire) et de Calcutta, les deux principales éditions arabes qui servent dorénavant de vulgates sans être parfaites pour autant. Leur traduction

ne prétend pas fournir l'image parfaite, exemplaire, des Nuits ; mais y en a-t-il une ? [...] la version de Bûlâq-Calcutta n'est ni plus ni moins sûre qu'une autre. Elle offre la suite intégrale des Nuits, de la première à la mille et unième, mais laisse sur la route divers textes que nous comptons légitimement au crédit et au trésor des Nuits, à commencer par ceux d'Aladin et d'Ali Baba ; il faudra donc nous les réapproprier en fin de recueil, comme une dernière retouche à ce grand corps réajusté. (Miquel 2005, XXXIX-XL)

Plus qu'à de la prudence philologique, cette analyse mène à reconsidérer la dépendance des Nuits vis-à-vis de la littérature arabe : le plaisir procuré par les Nuits peut « se partager dans toutes les langues du monde. Car [elles] appartiennent à la littérature universelle du conte, et nous ne découvrons que peu à peu leur apport exceptionnel au trésor de notre imaginaire à tous. " (Miquel 2005, XLV)

Prenant acte des métamorphoses travaillant les récits et les compilations, Jamel Eddine Bencheikh repense la notion d'œuvre, centrale pour cerner l'identité de ce qui ne constitue pas un texte mais assemble plusieurs manifestations textuelles, manuscrites et éditoriales : 


\begin{abstract}
Par œuvre, j'entends non pas une réalisation textuelle prise dans tel ou tel manuscrit, mais les mécanismes complexes, l'écheveau de significations, la totalité des représentations qui constituent l'existence profonde de ce corps vivant, sans cesse en activité féconde, en cours d'élaboration que sont les Nuits. Tout cela compte qui s'assemble, se pénètre et parle et se parle sans nécessité de lecteur, sans son existence même. Construction anonyme, travaillée par des siècles, s'originant en des mémoires lointaines, les Nuits ne peuvent mimer qu'elles-mêmes. (Bencheikh 1988, 15 ; en italiques dans le texte)
\end{abstract}

Bencheikh ne s'occupe donc ni du travail d'auteur ni de la réception de l'œuvre, mais de ce qui, de l'imaginaire collectif, se noue pour constituer l'œuvre puis passe et se perpétue de manifestation en manifestation, de texte en texte. Ce qui compte alors, c'est la manière dont chaque culture s'empare des récits ; et comment ces récits remettent à chaque fois en jeu des oppositions fondamentales et fondatrices de l'existence, entre le désir et la loi, autrement dit comment chaque récit participe de « [1]'aventure du sens dans un conflit des discours tenus par des antagonismes originels. Aventure du sens se démarquant des péripéties de l'anecdote. » (Bencheikh 1988, 11)

Il a poursuivi cette exploration de l'imaginaire par de nombreuses analyses des contes, puis du Voyage du prophète, ainsi que par une " conversation » avec André Miquel sur l'art, le savoir et le pouvoir dans la civilisation arabo-musulmane, c'est-à-dire plus précisément sur les rapports entre la conscience historique et l'imaginaire, la parole prophétique et la parole poétique, l'oral et l'écrit, entre l'État et son hétérogénéité ethnique et sociale, l'islamisme et les spiritualités hétérodoxes, la création et la violence, le voyageur et le monde qu'il arpente, l'amour et un art de vivre, questionnant l'unité de cette « civilisation ». Les deux chercheurs soulignent au passage l'hédonisme arabe exprimé par un discours sur le bien-vivre, un désir du plaisir tel qu'il se rêve par exemple dans Les Mille et Une Nuits. Tant pour la littérature du plaisir que pour le lien de l'écrivain au pouvoir, ils approfondissent une recherche esquissée dans les choix d'auteur et les remarques de René Khawam.

\title{
3 Une défense de l'imaginaire arabo-musulman
}

Poussé par ce même désir de faire comprendre la culture, méconnue ou bafouée, de l'Autre, en l'occurrence de sa communauté culturelle, René Khawam a rédigé un essai sur les Chrétiens d'Orient et des Contes d'Islam (1997) : ceux-ci mettent en scène le Prophète, ou ses compagnons, ou encore les premiers califes, dans des histoires honorant les valeurs de bravoure, de générosité, de loyauté. Bien qu'il n'indique aucune source, ses récits se rattachent à des traditions lettrées plus que populaires. 
Contribuer à une meilleure connaissance de la littérature arabe entre en tension, peu ou prou, avec les représentations des Européens à l'égard de la « civilisation arabe " et des relations géopolitiques de conflit et de domination qui ont saturé le $\mathrm{XX}^{\mathrm{e}}$ siècle. Pour l'un et l'autre, l'enjeu va donc bien au-delà de la mise à disposition des œuvres et de la visibilité de leurs auteurs. Il dépasse la connaissance d'une autre culture : il s'agit de combattre une méconnaissance de l'Autre dirigée par des traditions réductrices, externes ou internes au champ culturel arabe, que ce soit l'exotisme ou le dogmatisme.

En réaction aux doxas, Khawam vise à prouver l'intelligence et la profondeur de la culture arabe ; il met en valeur l' « état d'humanité » duquel la civilisation moderne de l'Occident serait désormais coupée (Hawrânî 1994, 10). Les textes sont présentés comme l'expression de « l'âme arabe » qui se distingue de l'esprit occidental par la prédilection de l'image sur le concept. Cet objectif justifie ses choix de traductions en poésie ainsi que le corpus choisi : la période abbasside où l'on " cultive des grâces et des impertinences nouvelles » (Khawam 1995, 22), célébrée jusqu’à la caricature : « On voudrait tout citer des œuvres de cette période où le génie arabe éclate comme jamais [...]. Autour de Charlemagne et de ses barons analphabètes, seule une poignée de moines sait lire et écrire ; au lieu qu'à Baghdâd, le premier savetier venu vous improvise à la demande un poème qui semble contenir tous les secrets de la terre et du ciel. " (Khawam 1995, 21). Il ne s'agit pas seulement de prouver la richesse littéraire arabe mais aussi de montrer à quel point ces discours littéraires, par leur idéologie, leurs valeurs, leur univers cognitif vont à l'encontre de bien des stéréotypes sur le monde arabo-musulman, en particulier de celui qui voudrait ne voir que l'hégémonie d'un Islam rigide. Khawam espère ainsi que Les Ruses des femmes, par sa sidérante singularité fasse «[...] voler en éclats quelques-unes des idées reçues les mieux ancrées que l'Occident persiste à entretenir à l'endroit de l’Islam [...]. » (Hawrânî 1994, 9)

Là où Khawam recueille des manifestations ponctuelles et singulières de « l'âme arabe », Jamel Eddine Bencheikh, quant à lui, s'intéresse moins à la force subversive d'un écrivain qu'à la différence entre une littérature dogmatique, occupant le centre du patrimoine littéraire, et une littérature de la marge, porteuse des désirs, des espoirs, d'un imaginaire libre, autrement dit des « discours » littéraires qui échappent aux injonctions idéologiques.

Plus intéressé par la circulation des récits ou de la « parole poétique », il se demande comment une légende, un mythe, un imaginaire, échappe à ses captations. Tandis que Khawam présente des auteurs à la fois érudits, consacrés, mais produisant des textes du plaisir, des livres que le « parfum de scandale » n'empêche pas d'avoir le statut de « best-sellers du Moyen Âge arabe » (al-Qâsîm alHarîrî 1992, 10), Bencheikh problématise cette coexistence à une échelle plus 
vaste. Il se demande comment, dans la narration, se mesurent désir et raison, comment s'établit une relation entre dogme et rêve. Autrement dit, il se demande plus concrètement ce qui fait qu'une légende parvient à s’insinuer dans des genres doctes de la raison.

\footnotetext{
Qu'est-ce qui fait, dans une culture arabo-islamique aussi fortement structurée, aux catégories si durement hiérarchisées, que la garde des marches soit impossible et, qu'une fois le corps étranger dans la place, on ne puisse le réduire ? Car le récit utopique s'évapore d'un lieu pour se recomposer ailleurs. Jamais on n'arrive à le dissoudre dans un discours. On peut l'inclure, non le digérer. Il rebondit de texte en texte en déjouant les surveillances. (Bencheikh 1990, 76)
}

Bencheikh a mené cette réflexion à partir des Mille et Une Nuits, de la légende d'Iram aux Colonnes et des récits de l'Ascension du Prophète. Dans ce dernier cas, sa réflexion s'est matérialisée par la publication en 1988 du Voyage nocturne de Mahomet à partir du Livre de l'Échelle. Il s'agit d'une tradition littéraire arabe (miraj) rapportant l'ascension du Prophète, en l'enrichissant de tous les compléments fournis par la littérature arabe. Malgré la source religieuse de l'histoire, il s'agit bien d'un imaginaire qui s'est bâti au-delà du Livre : " Le Coran n’a consacré qu'une ligne au voyage nocturne de Mouhammed : au-delà naît la légende. Celle-ci s'est constituée en textes qui ne sont légitimés que par l'imaginaire, et de cet imaginaire nous ne sommes pas sortis : notre composition avoue ses sources. » (Bencheikh 2002 [1988], 234)

Par l'exploration de l'imaginaire arabo-musulman, Bencheikh pointe la violence qui serait, selon lui, constitutive de l'histoire politique arabo-musulmane (Bencheikh et Miquel 1992, 33), même en ses périodes les plus glorieuses. Il en fait la tension cruciale de la narration des Nuits comme ce qui trame la lutte des discours et, par conséquent, la transmission de la mémoire.

\section{Khawam et Bencheikh écrivains : l’actualité de la parole}

Au seuil du Livre des ruses : la stratégie politique des Arabes, René Khawam affirme l'apport psychologique, historique et politique grâce auquel le lecteur, comme celui des Contes arabes d'Antoine Galland près de trois siècles plus tôt, peut mieux comprendre le monde arabe (Ruses 1976, 15). Une telle actualité du texte s'explique, selon lui, par l'identité civilisationnelle d'hier à aujourd'hui : «la pensée et la mentalité musulmanes ne diffèrent pas grandement aujourd'hui de ce qu'elles furent il y a des siècles - ce qui fait d'ailleurs la vraie force de l'Islam 
face à un monde dont les valeurs apparaissent chaque jour plus incertaines. » (Ruses 1976, 10) La continuité de mentalité tiendrait à la préservation de la langue arabe, à son sémantisme, aux représentations qu'elle véhicule. En effet, si l'on « accède » un tant soit peu à la « vérité » d'un univers culturel, c'est par les signes de la langue : «Car s'il n'est pas mauvais d'avoir franchi du bon pied le porche du politique, on ne saurait comprendre le fonctionnement du monde qui s'ouvre derrière lui si l'on n'en a pas arpenté les ruelles, les passages secrets, les lieux de perdition - et moins encore si l'on n'a pas goûté la magie opératoire du langage qui y a cours. » (al-Qâsîm al-Harîrî 1994, 13) Le franchissement semble rester le privilège du traducteur, le lecteur, faute d'outil, restant au seuil. Il ne le passera qu'avec les créations - contes et poésie - des deux auteurs.

René Khawam a composé deux recueils de contes qui décevraient celui qui s'attendrait à des pièces de tradition orale. L'un, intitulé Contes d'Islam, contient des récits repris à des traditions islamiques ; l'autre, Contes $d u$ Liban regroupe des textes renvoyant à un univers populaire mais dans un style qui ne cherche pas à imiter l'oralité. Ces histoires, parfois truculentes, souvent malicieuses, racontent les balbutiements du désir et ses mirages, la victoire des simples et de leurs vertus - dans la lignée des contes populaires. Ni « édifiantes » ni sociologiques, elles donnent à voir des intrigues où la parole est un acteur essentiel, source de conflits, de solutions, de malentendus, de mystères. La verve de l'auteur fait vivre le réel et les rêves, les désirs et les normes d'un univers - celui de la communauté chrétienne libanaise - rarement représenté dans la littérature française.

Dans aucun de ces deux recueils, les sources ne sont mentionnées, si bien qu'on ne sait s'il faut y voir une création d'auteur ou une compilation ${ }^{4}$. Ces récits ne correspondent ni à une littérature lettrée ni à une transcription de textes populaires. L'affichage du genre « conte » oriente cependant la lecture plutôt dans le sens d'une parole collective dont le texte constitue un relais.

Compilateur d'une Anthologie de la nouvelle poésie algérienne, Bencheikh est également l'auteur d'une œuvre poétique féconde, étalée dans le temps. Alors que dans ses études sur les écritures de l'imaginaire, le poéticien s'attache moins à la manipulation du langage qu'à la « magie opératoire » du récit, ses modalités de significations ou sa charge socioculturelle, il explore les ressources de la langue - française - au fil de plusieurs recueils de poésie dont les publications se sont échelonnées sur deux décennies.

4 Néanmoins, on suppose que dans le second cas, la patte de l'auteur est bien plus marquée que dans le premier, où Khawam suggère une tradition remontant à des témoins qu'il mentionne. 
Son écriture en français, langue « insolite et familière », si elle se justifie par une « contrainte historique ", ne tourne pas le dos à la langue arabe ${ }^{5}$, qui est à la fois celle du Coran récité par son père et la variante dialectale parlée par sa mère. Si le contexte référentiel est souvent générique, assez peu rattaché à des situations sociohistoriques, l'écrivain introduit parfois dans la langue d'écriture un imaginaire venu d'ailleurs, difficilement perceptible pour qui ne partage pas ces références ${ }^{6}$.

Comme imprégnée des questionnements de la modernité poétique du XX $\mathrm{X}^{\mathrm{e}}$ siècle quant au sujet du poème, cette poésie creuse les cheminements intérieurs de l'être par lesquels s'énonce un « je » élaboré dans et par le poème. Au cours cette quête, le poète dit souvent son face à face avec les constituants et la fabrication du poème. Cette fabrication, matérialisée par des images à valeur « existentielle » renvoie au travail physique agi et subi ${ }^{7}$, à la tension entre sensible et impalpable. Elle laisse entendre la fragilité du désir, elle place sous un permanent sursis la vitalité de la parole. Dès lors que l'on prend en compte, dans la mise en abîme de la création, l'achèvement des textes tantôt sur la désillusion du poète et le mutisme du poème, tantôt sur l'espérance, on est tenté de considérer que Bencheikh " poétise » l'étreinte batailleuse de la vie et de la mort, du désir et de la loi, telle qu'il l'analyse dans Les Mille et Une Nuits. Preuve d'une persistance de ce conflit, plusieurs poèmes se réclament du conte, comme si un lien s'établissait au-delà de la distance temporelle et de l'écart formel pour encore et toujours animer une voix contre la violence.

\section{Conclusion : pour le ludisme}

Jamel Eddine Bencheikh et René Rizqallah Khawam ont mis chacun leur double culture arabe et française au bénéfice d'une meilleure connaissance de la littérature et de la civilisation arabo-musulmanes. Si, jusqu'à la moitié du XXe siècle, les orientalistes européens avaient la mainmise sur leur transmission en Europe, les

5 «Ce n'est ni une identité ni un exil que je cherchais dans le français, mais la certitude d'y être libre et solitaire [...]. Seule la poésie me semblait capable de libérer mon langage et son inaltérable prétention à reprendre sans cesse ses périples. » (Bencheikh 1999, 64-65).

6 Ainsi du poème "Ainsi parla Shahrazad », ou de celui sur Kateb Yacine dans Cantate pour le pays des îles (1997, 17-23, 63-73).

7 Cette poésie, qui se refuse à taire les meurtrissures, nomme l'entaille et la faille, le démembrement et le creusement, tout en rêvant un tissage que le poème continue à réaliser. La tension entre la blessure et la persistance à nommer se perçoit plus à l'échelle du recueil qu'à celui du poème, rarement éclaté. 
deux arabisants présentés ici l'ont infléchie grâce à une série de questionnements historiques, sociologiques et génétiques ; grâce, également, à l'extériorité à la tradition linguistique et philologique française instaurée par leur culture arabe ${ }^{8}$. D’autre part, ils ont incité à s'aventurer vers d'autres textes que les grands classiques à une époque où l'édition de traductions de la littérature arabe commençait seulement à s'accroître, s'appuyant encore sur une poignée d'auteurs et traducteurs ${ }^{9}$.

En dépit de leurs divergences dans l'approche des œuvres, ils ont partagé, l'un par ses études sur les textes et son regard sur l'actualité politique et culturelle, l'autre par ses traductions nombreuses et partisanes, la conscience de la charge idéologique de ces textes, subversifs en ce qu'ils proposent d'autres voix que celles du dogme - qu'il soit arabe ou français, islamique ou fantasmé. Avec d'autres arabisants de la fin du $\mathrm{XX}^{\mathrm{e}}$ siècle, ils avaient en commun la volonté d'agir - pour les modifier - sur les points de vue stéréotypés ${ }^{10}$, exotiques, sur la littérature et la culture arabes : «Par là on voit bien la nécessité de corriger la représentation que l'on donne des Arabes encagés dans l'islam, présentant un moralisme agressif et un fatalisme abêtissant. Cette civilisation comporte au contraire une formidable pulsion ludique. On aime s'assembler, chanter, boire, faire de la musique, plaisanter. » (Bencheikh et Miquel 1992, 168). C'est sans doute un apport majeur de ces deux passeurs que d'avoir donné à lire, à ressentir et à penser cet élan ludique, sans idéalisme pour autant, mais au contraire en le faisant valoir, aux yeux non seulement des Français mais aussi des Arabes, avec et contre ce qui le nie.

8 Ils correspondent au profil des migrants intellectuels dont Thomas Brisson, dans Les Intellectuels arabes en France, analyse la place et l'influence : « Originaires du monde arabe, ils en possèdent la culture lettrée et la langue ; mais ils sont en même temps chercheurs ou universitaires intégrés dans les structures de savoir françaises et dans les rationalités scientifiques occidentales. Intellectuels de deux mondes, ils forment l'un des vecteurs de communication entre savoirs européens et arabes, permettant de saisir comment ces savoirs dialoguent, s'opposent et se mélangent. » (Brisson 2008, 8).

9 Nada Tomiche a établi qu'entre la fin de la Seconde Guerre mondiale et le début des années 1970, la majorité de la littérature arabe traduite concernait majoritairement les contes des Mille et une nuits et quelques grands classiques (Tomiche 1978).

10 Voir la réflexion de Christiane Chaulet Achour (2013) sur le travail de subversion du regard orientaliste par ces arabisants, universitaires français de culture arabe. Elle rattache leur relecture des Mille et Une Nuits à la remise en cause du discours orientaliste par Edward Saïd. 


\section{Bibliographie}

al-Qâsîm al-Harîrî. Le Livre des Malins : séances d'un vagabond de génie. René Khawam (trad.). Paris : Phébus, 1994.

al-Souyoûti, Abd al-Rahmane. Nuits de noces, ou comment humer le doux breuvage de la magie licite. René Khawam (trad.). Paris : A. Michel, 1972.

Bencheikh, Jamel Eddine. Poétique arabe : essai sur les voies d'une création. Paris : Éditions Anthropos, 1975.

Bencheikh, Jamel Eddine. Les Mille et Une Nuits ou la parole prisonnière, Paris : Gallimard, «NRF », 1988.

Bencheikh, Jamel Eddine. «Iram ou la clameur de Dieu. Le mythe et le verset». Revue du monde musulman et de la Méditerranée 58 (1990):70-81.

Bencheikh, Jamel Eddine. Cantate pour le pays des îles. Paris : Marsa, 1997.

Bencheikh, Jamel Eddine. Failles fertiles du poème. Saint-Benoît-du-Sault : Tarabuste, 1999.

Bencheikh, Jamel Eddine. Le Voyage nocturne de Mahomet, suivi de L'Aventure de la parole.

Paris : Imprimerie Nationale Éditions, 2002 [1988].

Bencheikh, Jamel Eddine, et André Miquel. D'Arabie et d'Islam. Paris : O. Jacob, 1992.

Brisson, Thomas. Les Intellectuels arabes en France. Paris : La Dispute, 2008.

Chaulet Achour, Christiane. Jamel Eddine Bencheikh. Une parole vive. Montpellier : Chèvrefeuille Étoilé, 2006.

Chaulet Achour, Christiane. "Orientalisme et champ universitaire français au XXe siècle Les Mille et une nuits ". Les Orientaux face aux orientalismes. Ridha Boulaâbi (dir.). Paris : Geuthner, 2013. 87-107.

Hawrânî, Abd al-Rahîm. Les Ruses des femmes. René Khawam (trad.). Paris : Phébus, 1994.

Ibn al-Muqaffa', 'Abdallah. Le Pouvoir et les intellectuels ou les aventures de Kalîla et Dîmna. René R. Khawam (trad.). Paris : Maisonneuve et Larose, 1985.

Khadda, Naget. "L'île enchantée ». Cahiers Jamel Eddine Bencheikh : savoir et imaginaire. Christiane Chaulet Achour (dir.). Paris et Montréal : L'Harmattan, 1998. 23-43.

Khawam, René. La Poésie arabe : des origines à nos jours. Paris : Phébus, 1995 [1977].

Khawam, René. Contes du Liban. Paris : Éditions Asfar, 1989.

Khawam, René. Contes d'Islam. Paris : L'Esprit des Péninsules, 1998.

Miquel, André. « Préface ». Les Mille et Une Nuits. Vol. 1. André Miquel et Jamel Eddine Bencheikh (trad.). Paris : Gallimard, « Bibliothèque de la Pléiade », 2005.

Nawâjî, Muhammad Ibn Hasan, La Prairie des gazelles : éloge des beaux adolescents. René R. Khawam (trad.). Paris : Phébus, 1989.

[Ruses]. Le Livre des ruses : la stratégie politique des arabes. René Khawam (trad.). Paris : Phébus, 1976.

Tomiche, Nada. La Littérature arabe traduite. Paris : Geuthner, 1978.

Cyrille François holds a doctorate in French, Francophone and Comparative Literatures (University of Cergy-Pontoise, France) with a dissertation about The Thousand and One Nights in Modern Literature. He his the author of books on Da- 
niel Maximin's L'Isolé Soleil and Aimé Césaire's Le Cahier d'un retour au pays natal. He has edited Le don de Shahrâzâd : la mémoire des Mille et Une Nuits dans la littérature contemporaine (2008) and (with Corinne Blanchaud) Pour la poésie : Poètes de langue française (XXe-XXIe siècles) in 2016. His current research focuses on modern poetry and tales. 